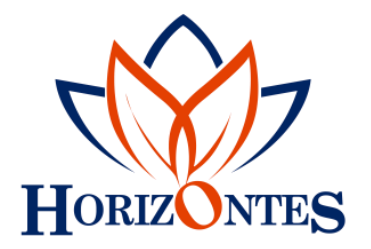

Horizontes. Revista de Investigación en Ciencias de la Educación Https://doi.org/10.33996/revistahorizontes.v5i18.181

Abril - junio 2021

Volumen 5 / No. 18

ISSN: 2616 - 7964

ISSN-L: $2616-7964$

pp. $382-390$

www.revistahorizontes.org

\title{
Geogebra: herramienta tecnológica para el aprendizaje significativo de las matemáticas en universitarios
}

\author{
Geogebra: technological tool for the meaningful learning of mathematics in university \\ students
}

\section{Geogebra: ferramenta tecnológica para a aprendizagem significativa da matemática em estudantes universitários}

\section{ARTÍCULO DE REVISIÓN}

\author{
Fernando Ysmael Cenas Chacón \\ fernando.cenas@upn.edu.pe \\ https://orcid.org/0000-0003-0237-0737 \\ Leydidiana Rosibel Gamboa Ferrer \\ leydidiana.gamboa@upn.edu.pe \\ https://orcid.org/0000-0002-9393-4202
}

\author{
Fanny Evelyn Blaz Fernández \\ fanny.blaz@upn.edu.pe \\ https://orcid.org/0000-0002-1390-3810 \\ Wilmer Edwin Castro Mendocilla \\ wilmer.castro@unas.edu.pe \\ https://orcid.org/0000-0002-8719-237X
}

Universidad Nacional de Trujillo, Trujillo-Perú

Recibido 08 de enero 2021 | Arbitrado y aceptado 15 de febrero 2021| Publicado en 01 abril 2021

\section{RESUMEN}

La educación se ha visto en la necesidad de hacer posible que los procesos de enseñanza-aprendizaje estén acordes a la era digital. En este sentido, el objetivo general de esta investigación fue reflexionar sobre la influencia que el software GeoGebra tiene en el aprendizaje significativo en las matemáticas en estudiantes universitarios. La metodología utilizada se basó en una revisión sistemática con un nivel descriptivo cualitativo. Como resultados se describió la importancia del aprendizaje significativo, el rol de las TIC en la educación, el TIC en la etapa universitaria, herramientas tecnológicas en las matemáticas, y las características de GeoGebra. Como conclusión, la utilidad de GeoGebra permite al estudiante analizar de manera más detallada contenidos matemáticos, en menor tiempo, así como también alcanzar objetivos claves como la mejora del rendimiento académico y el desarrollo pleno de las competencias matemáticas para su posterior puesta en práctica cuando el alumno sea un profesional.

Palabras clave: GeoGebra; aprendizaje significativo; matemáticas

\section{ABSTRACT}

Education has seen the need to make it possible for the teaching-learning processes to be in line with the digital age. In this sense, the general objective of this research was to reflect on the influence that GeoGebra software has on meaningful learning in mathematics in university students. The methodology used was based on a systematic review with a qualitative descriptive level. As results, the importance of meaningful learning, the role of ICT in education, ICT in the university stage, technological tools in mathematics, and the characteristics of GeoGebra were described. In conclusion, the utility of GeoGebra allows the student to analyze mathematical contents in a more detailed way, in less time, as well as to achieve key objectives such as the improvement of academic performance and the full development of mathematical competencies for their subsequent implementation when the student be a professional.

Key words: GeoGebra; meaningful learning; mathematics
RESUMO

A educação tem visto a necessidade de possibilitar que os processos de ensinoaprendizagem estejam em consonância com a era digital. Nesse sentido, o objetivo geral desta pesquisa foi refletir sobre a influência que o software GeoGebra exerce na aprendizagem significativa em matemática em estudantes universitários. A metodologia utilizada baseou-se em revisão sistemática com nível descritivo qualitativo. Como resultados, descreveu-se a importância da aprendizagem significativa, o papel das TIC na educação, as TIC na fase universitária, as ferramentas tecnológicas da matemática e as características do GeoGebra. Concluindo, a utilidade do GeoGebra permite ao aluno analisar conteúdos matemáticos de forma mais detalhada, em menos tempo, bem como atingir objetivos fundamentais como a melhoria do desempenho acadêmico e o pleno desenvolvimento de competências matemáticas para sua posterior implementação quando o aluno seja um profissional.

Palavras-chave: GeoGebra; aprendizagem significativa; matemática 


\section{INTRODUCCIÓN}

Durante los últimos diez años, las sociedades han sufrido cambios debido al desarrollo y difusión de nuevas tecnologías de información y comunicación, posteriormente conocidas por sus siglas TIC, las mismas que se desenvuelven en entornos digitales cuyo elemento principal es el internet (Suasnabas, Díaz, Ávila, \& Rodríguez, 2017). En ese sentido, el surgimiento y desarrollo del internet, según Islas, (2017), representó la reestructuración de las actividades que la mayoría de los humanos realiza multiplicando las posibilidades de acceso a la información, transacciones comerciales y por supuesto también implicó cambios en la educación.

De esta forma, la educación se ha visto en la necesidad de hacer posible que los procesos de enseñanza-aprendizaje estén acordes a la era digital, sometiéndolos a una acelerada adaptación a los avances, implicando la aplicación de metodologías de formación adecuadas en las que se priorizaba el equilibrio entre la información, el conocimiento, la comunicación y la producción del mismo conocimiento Islas, (2017). Las TIC, así, imponen a las instituciones educacionales, en particular a las de educación superior, la necesidad de realizar transformaciones en sus procesos formativos, de modo que estos respondan a las actuales circunstancias del contexto social donde se desenvuelven (Zambrano \& Zambrano, 2019).

En ese sentido, las matemáticas son un tema de real importancia siendo a través de ella que se puede conocer e interpretar el mundo, así como tomar decisiones. Sin embargo, a lo largo de los años ha existido aversión y desmotivación de los estudiantes hacia ellas Tamayo, (2013), conllevando a la repetición o abandono académico por no superarlas. Esta situación, en la actualidad ha cambiado
(Mendoza, et al, 2014; Pabón, Nieto, y Gómez, 2015). De manera específica, los productos tecnológicos han influenciado en la manera de aprender y enseñar las matemáticas, en la medida que generan imágenes, organizan $\mathrm{y}$ analizan datos y presentan diversos recursos y herramientas que favorecen esta compleja disciplina (Sánchez, 2012).

Una de estas herramientas es el software GeoGebra el cual es considerado como instrumento de aprendizaje que brinda la oportunidad para transformar el ambiente tradicional del sistema educativo, en un espacio de interacción que conlleva al estudio comprensivo de las matemáticas y al desarrollo de las capacidades que le permiten adquirir un aprendizaje significativo (Pabón et al. 2015).

Por todo lo expuesto, el presente estudio se planteó como objetivo general revisar la utilidad como herramienta tecnológica del software GeoGebra, en el aprendizaje significativo de las matemáticas en estudiantes universitarios.

\section{METODOLOGÍA}

Esta investigación consistió en una revisión sistemática de artículos científicos indexados ubicados en las páginas de búsqueda de información científica como Proquest, Scopus y Google Académico. Luego, se procedió a realizar la búsqueda a través de palabras clave que orienten en los documentos científicos necesarios. Estas corresponden a "GeoGebra", "matemáticas", "aprendizaje significativo" y "estudiantes universitarios". Se combinaron entre si los descriptores y durante la búsqueda se usaron los operadores boléanos "and" y "or". Además, algunos de los criterios o filtros que se utilizaron para hacer muchos más específica la búsqueda y ser más precisos con los documentos encontrados en las diferentes 
bases de datos consultadas fueron: que sean artículos de revistas, y la antigüedad de la publicación no mayor a 10 años, procurando que la mayor parte de las fuentes se mantenga en 5 años atrás. Esto arrojó como resultado 21 documentos científicos que fueron tomados en cuenta para el desarrollo de la presente investigación.

Toda la información previa relacionada a los filtros aplicados y las especificaciones sirvieron como criterios de inclusión, es decir que sean artículos publicados en revistas científicas, que no excedan los cinco años de antigüedad, que la temática guarde relación con las variables de GeoGebra y aprendizaje significativo de matemáticas. Se excluyeron, en tanto, todos los documentos que estuvieran orientados a estudiantes de otros niveles de educación, que no respetaran las especificaciones postuladas, así como aquellos que estaban incompletos o que tenían enlaces averiados.

El desarrollo de la información se realizó de forma sistemática, partiendo de la importancia de las estrategias de enseñanza, procesos y estilos de aprendizaje, estrategias de enseñanza de la matemática, educación virtual de las matemáticas, plataformas virtuales para la enseñanza de las matemáticas y la plataforma GeoGebra como herramienta generadora de conocimientos.

\section{DESARROLLO Y DISCUSIÓN}

\section{Aprendizaje significativo}

El aprendizaje significativo, según Moreira (2019) supone un enfoque teórico que establece que el principal protagonista en la educación es el estudiante, el mismo que construye su conocimiento haciéndolo parte de su esquema cognoscitivo a través de un proceso de aprendizaje dinámico y autocrítico.

En el aprendizaje significativo hay una interacción clarificadora y amplificadora entre el nuevo conocimiento y los ya existentes, en la cual ambos se modifican y enriquecen en la medida en que el conocimiento inicial contribuye a asignar significado al nuevo y de esta manera él también se enriquece y amplía (Latorre, 2017).

Al respecto, Ausubel (1978) citado por Gómez, Muriel, y Londoño, (2019) señala que los docentes deben diseñar las estrategias didácticas que le permitan relacionar las experiencias previas y los nuevos aprendizajes que se proponen a partir de las necesidades, interés y problemas del estudiante. No obstante, este proceso ha evolucionado al punto que se considera la apropiación y dominio que tiene el docente y estudiante con la tecnología, por lo tanto se presenta en el proceso de aprendizaje otro factor como lo es el manejo de los recursos tecnológicos (Suárez, Vélez, y Londoño, 2018).

Jiménez, Vico, y Rebollo, (2017) mencionan, al respecto, que las TIC ofrecen una gran cantidad de posibilidades para el diseño de nuevos espacios de aprendizaje, situando a los estudiantes en el centro del proceso de aprendizaje, siendo este, como se ha mencionado, el fundamento del aprendizaje significativo.

\section{El rol de las TIC en la educación}

Serrano et al. (2016) citado en Torres y Cobo, (2017) afirman que la tecnología en la educación, o como los autores han denominado 'tecnología educativa' constituye una disciplina encargada del estudio de los medios, materiales, portales web y plataformas tecnológicas al servicio de los procesos de aprendizaje. 
Como se mencionó previamente, en los ámbitos educativos, de manera específica en el aprendizaje significativo se requiere, en la actualidad, la implementación de las TIC para actualizar y dinamizar el conocimiento (Moreira, 2019).
Esto ha hecho que se generen cambios en el quehacer docente y también en el rol del alumno tal como se muestra en la Tabla 1.

Tabla 1. Cambios en los roles de docentes y alumnos orientado a un aprendizaje significativo

\section{ENTORNO DE APRENDIZAJE CENTRADO EN EL DOCENTE Y CENTRADO EN EL ALUMNO \\ ANTES \\ DOCENTE \\ HOY \\ DOCENTE}

Transmisor de conocimientos, de información, Guía del aprendizaje, colaborador, tutor y facilitador poseedor de la verdad y todas las respuestas en el proceso enseñanza-aprendizaje. Como evaluador identifica errores, refuerza aciertos, realiza comentarios pertinentes, señala criterios de trabajo, actuación entre otros.

Controla y dirige todos los contenidos del Permite que los alumnos sean más responsables de su aprendizaje

ESTUDIANTE

Receptor pasivo de la información

Reproductor del conocimiento

El aprendizaje es una actividad individual
ESTUDIANTE propio aprendizaje

Participante activo del proceso de aprendizaje

Produce y comparte el conocimiento de forma más participativa y abierta

El aprendizaje es una actividad colaborativa que se realiza con otros estudiantes

Fuente: (García, Marbán, \& Arnal, 2020)

Ahora bien, Zambrano y Zambrano (2019) citan a Vaquero (1995) para exponer una definición de las TIC en la educación y plantea que son "las propuestas electrónicocomunicativas que organizan el entorno pedagógico diseñando propuestas educativas interactivas y que trascienden los contextos físicos, fijos, institucionales, a fin de hacerlos asequibles a cualquiera, en cualquier tiempo $\mathrm{y}$ lugar" (p.5).

Así, la incorporación de las TIC a la educación se ha convertido en un proceso que implica no solo el uso de herramientas tecnológicas sino un cambio en la construcción didáctica y la manera cómo se pueda construir y consolidar un aprendizaje significativo en base a la tecnología (Díaz, 2013).

\section{TIC en la etapa universitaria}

Las TIC son un medio con influencias educativas para diversos niveles incluyendo la formación profesional de los estudiantes de las múltiples carreras universitarias. El efecto de las TIC en la educación superior, según Zambrano y Zambrano (2019) se manifiesta de manera muy especial, pues su alcance va desde la estructura y objetivos generales de la universidad, hasta aquel lugar donde, profesores y estudiantes se encuentran en el acto de enseñar y aprender. 
Las TIC en la educación superior representan los nuevos entornos de aprendizaje y, por su impacto en la educación, desarrollan las competencias necesarias para el aprendizaje y habilidades para la vida. Según García et al. 2020,las TIC en la etapa universitaria suponen un apoyo en la interacción con actividades didácticas que integran lo visual, novedoso e interactivo; incentivan el uso de aplicaciones, plataformas y redes sociales; promueven nuevas formas de enseñanza; facilitan la búsqueda de información y comunicación, y el desarrollo de actividades prácticas del quehacer docente.

Los autores indican que, debido a las TIC, los docentes tienen el deber de hacer uso de todas las variedades de alternativas tecnológicas que se presentan para con ellas despertar interés en los contenidos de las unidades de aprendizaje y generar nuevas actitudes entre los estudiantes. En ese sentido, esto es un beneficio sin precedentes para el cambio en una disciplina compleja como las matemáticas.

\section{Herramientas tecnológicas en las matemáticas}

La visión clásica del proceso enseñanzaaprendizaje de la matemática, según Godino, Batanero y Font (2003) citados en Bravo, Arenas, y Pineda, (2019) se define como un intercambio entre profesor y alumnos donde el primero tiene protagonismo, mientras que el estudiante asume un rol pasivo.

Sin embargo, desde la concepción del aprendizaje significativo y según detalla Moreria, (2017) el profesor tiene un rol de mediador, dado que el domina los significados y busca ser el vínculo para entregárselos al estudiante. El alumno, en tanto, tiene también el trabajo de buscar y captar tales significados. El profesor presenta de diversas formas los conocimientos pero al estudiante le compete verificarlos.

Se debe sumar a ello, el rol de las TIC que, como se sabe, han cambiado el mundo de las matemáticas y el de las ciencias, respecto a cómo se hacen, se enseñan, se construyen, se profundizan, se aprenden y se transmiten como legado (Bravo,et al 2019).

Por ello, se debe relacionar en el proceso de aprendizaje significativo de las matemáticas el contexto de la tecnología y la utilización de un lenguaje pertinente, ello implica que los docentes definan estrategias pedagógicas y didácticas que se caractericen por ser innovadoras, quesean atractivas para los alumnos logrando generar motivación e interés por aprender, antes que de solo informarse (Díaz y Omara, 2014 citado en Gómez et al. 2019).

Los productos tecnológicos, así, van a cambiarlos entornos educativos y ello incluye las formas de aprender la matemática. Uno de los procesos que permiten las TIC en el aprendizaje de la matemática, es la comprensión y descubrimiento de nociones a través de la visualización de imágenes y gráficos, así como mediante la organización y análisis de datos, posibilitando, finalmente, que el estudiante se centre en procesos relacionados a la toma de decisiones, la reflexión, el razonamiento y la resolución de problemas. Afianzando lo anteriormente descrito, Bravo et al (2019), precisan que en este contexto, el uso de software para aprender matemáticas debe ser un elemento clave.

Los softwares educativos orientados al área matemática pueden ser herramientas didácticas que le permiten al docente proponer situaciones de enseñanza que modifican las interacciones en el aula, generan espacios 
diferentes para explorar, conjeturar y demostrar propiedades de la aritmética, del álgebra, de la geometría y del análisis (Natale \& Papini, 2019).

El software para la enseñanza matemática es uno de los recursos más poderosos que la tecnología ha brindado a las ciencias matemáticas, contribuyendo en su aprendizaje, tal es así como los programas MatLab, CabriGeometre, Derive,Wímplot y GeoGebra (Hernández y Revilla, 2017; Valderrama y Saldaña, 2020).Esta investigación se ha basado específicamente en el GeoGebra que es un programa informático dinámico diseñado para la enseñanza-aprendizaje de las matemáticas, y que es usado sin mayores complicaciones por los estudiantes.

\section{GeoGebra}

GeoGebra es un programa ideado como herramienta didáctica, permite la búsqueda y la investigación como medios para aprender matemática. Es una herramienta tecnológica que abre la posibilidad de abordar problemas que serían imposibles sin su ayuda y permite adoptar un enfoque experimental de la matemática cambiando la naturaleza de su aprendizaje (Natale \& Papini, 2019).

Dentro del universo de softwares educativos, GeoGebra ha sido el que ha ido haciéndose paso por encima de todos los demás. Esto según explica García et al. (2020) por su facilidad y conexión, además de poder realizar construcciones dinámicas e interactivas, facilitando así su implantación en el aula.

El software GeoGebra inicialmente fue diseñado para la enseñanza y el aprendizaje de la geometría y el álgebra, de ahí su nombre, sin embargo, en el año 2009, tal y como explica García, et al. (2020), se introdujeron comandos de funciones estadísticas y gráficos y en el año 2011 el análisis de datos y el cálculo de probabilidades. La incorporación de estas herramientas y una interfaz pensada para los estudiantes, de fácil manejo, son otras de las razones que lo hacen más atractivo en comparación con otros softwares.

Específicamente, Hernández y Revilla, (2017) ejemplificaron en su investigación, las ventajas y utilidad del software GeoGebra por medio de consideraciones didácticas y ejercicios en carrearas universitarias de Ingeniería y Arquitectura, lo que permitió ilustrar el mejoramiento de la enseñanza- aprendizaje de la matemática. Así mismo, comentan que GeoGebra es un software interactivo que integra de manera dinámica aspectos o contenidos de diversas áreas de la matemática y es importante ya que facilita los cálculos y visibilidad de graficas además de estimular la creatividad de los estudiantes y los docentes.

Lo relevante de la investigación anterior es que la propuesta de consideraciones didácticas y ejercicios, fueron evaluados favorablemente entre estudiantes y docentes, en conferencias, espacios de preparaciones metodológicas y consultas ofrecidas por los autores. Sin embargo, estas evaluaciones también reflejaron insuficiencias presentes en los estudiantes en la construcción del planteamiento y análisis de las soluciones de ejercicios. (Hernández y Revilla, 2017).

Valderrama y Saldaña (2020) en tanto, demostraron que utilizar el programa GeoGebra les proporciona a los estudiantes visualizar de forma rápida los diferentes lugares geométricos que se presentan en el estudio de la geometría analítica plana como la recta, circunferencia, la parábola entre otras figuras. Los autores aseguraron que existe una influencia significativa del software GeoGebra en el 
rendimiento académico de los estudiantes en el curso de complemento matemático.

En esa línea, analizando los resultados de Zenteno, Rivera, y Pariona, (2020) en la investigación cuyo objetivo fue explicar el aprendizaje de las medidas de dispersión por medio del Software GeoGebra en los estudiantes del Laboratorio de Investigación e Innovación Pedagógica de la Universidad Nacional Daniel Alcides Carrión de Pasco Perú-2017, se llega a la conclusión que la aplicación del software GeoGebra a los estudiantes del Laboratorio de Innovación Pedagógica del grupo experimental tienen mejores resultados que del grupo de control, comprobando que el manejo de esta aplicación educativa mejoraba la enseñanzaaprendizaje y el rendimiento académico.

\section{CONCLUSIONES}

Las TIC representan a las herramientas de trabajo que hacen posible la integración de diferentes medios con las correspondientes ventajas de cada uno, favoreciendo la trasmisión de más información en menos tiempo, de forma variada $\mathrm{y}$ amena $\mathrm{y}$ permitiendo el establecimiento de un ambiente de aprendizaje favorable y significativo donde se siente motivado el estudiante gracias a su carácter de interactividad.

Asimismo, debido a las necesidades de la sociedad actual, se busca mostrar al estudiante la gran cantidad de habilidades que tiene y cómo con las tecnologías digitales se ve favorecido para desarrollarlas y plasmarlas como un bien social.

El mejoramiento de la enseñanzaaprendizaje de la matemática en carreras universitarias es fundamental y por eso es importante el acceso a internet y software gratuitos que promuevan el desarrollo del razonamiento matemático de los estudiantes.
La utilidad de las TIC en general $\mathrm{y}$ GeoGebra en particular, es que le permite al alumno analizar de manera más detallada contenidos matemáticos, en menor tiempo, así como también alcanzar objetivos claves como la mejora del rendimiento académico y el desarrollo pleno de las competencias matemáticas para su posterior puesta en práctica cuando el alumno sea un profesional.

GeoGebra es un software sencillo de usar y que, sin embargo, tiene grandes beneficios para los estudiantes universitarios en la disciplina de las matemáticas. La revisión bibliográfica ha permitido observar cómo, desde que las TIC se introdujeron en la educación y forma específica en la educación superior, es decir en las aulas de estudiantes universitarios, han tenido gran repercusión en los métodos de enseñanzaaprendizaje; teniendo, sobre todo, un rol importante en el aprendizaje significativo.

Otra de las utilidades del GeoGebra es que promueve análisis a ejercicios, vías de solución $\mathrm{y}$ tratamiento didáctico que favorecen $\mathrm{y}$ estimulan la creatividad ya que ilustra de manera visual los contenidos matemáticos de los universitarios. Por todo esto se recomienda incorporar en el aprendizaje de las ciencias y en las aplicaciones prácticas el uso de herramientas tecnológicas como el GeoGebra.

Finalmente, el Programa Aplicativo o software GeoGebra es considerado como una estrategia didáctica para mejorar el rendimiento académico por su aporte al aprendizaje significativo.

\section{REFERENCIAS}

Bravo, A., Arenas, J., y Pineda, E. (2019). El aprendizaje de la geometría con GeoGebra, un enfoque de aprendizaje por problemas. . Revista Docencia Universitaria, 20(2), 55-67 
Díaz, F. (2013). TIC en el trabajo del aula. Impacto en la planeación didáctica. . Revista Iberoamericana de Educación Superior, 4(10), 3-21

García, Y., Marbán, M., y Arnal, M. (2020). Percepción de los estudiantes sobre el software GeoGebra en el estudio de la estadística en los grados de Educación. XXVIII Jornadas ASEPUMA - XVI, Encuentro Internacional Anuales de ASEPUMA nํ28: A105

Gómez, L., Muriel, L., y Londoño, D. (2019). El papel del docente para el logro de un aprendizaje significativo apoyado en las TIC. . Encuentros, 17(2), 118-131

Hernández, C., y Revilla, A. (2017). Utilización del GeoGebra en el primer año de carreras universitarias: Ejemplos y consideraciones didácticas. Tecnología Educativa, 2(1), 3948

Islas, C. (2017). La implicación de las TIC en la educación: Alcances, Limitaciones y Prospectiva. . RIDE, Revista Iberoamericana para la Investigación y el Desarrollo Educativo, 8(15)

Jiménez, R., Vico, A., y Rebollo, Á. (2017). Female university student's ICT learning strategies and their influence on digital competence. . International Journal of Educational Technology in Higher Education, (14), 34

Latorre, M. (2017). Aprendizaje Significativo y Funcional. Informe de la Universidad Marcelino Champagnat

Mendoza, U., Candella, R., Assad, L., Castillo, F., Azevedo, L., Knoppers, B., y Albuquerque, A. (2014). A Model Analysis for the Design and Deployment of an Eulerian Sediment Trap Mooring Array in a Western Boundary Upwelling System from Southeast Brazil. Anais da Academia Brasileira de Ciências, 86(2), 589-600

Moreira, P. (2019). Las TIC en el aprendizaje significativo y su rol en el desarrollo cognitivo de los adolescentes. ReHuSo: Revista de Ciencias Humanísticas y Sociales, 4(2)., 1-12
Moreria, M. (2017). Aprendizaje significativo como un referente para la organización de la enseñanza. Archivos de Ciencias de la Educación, 11(12)

Natale, M., y Papini, M. (2019). Producir geometría con GeoGebra. Una experiencia colaborativa en el nivel universitario. Argentina: Actas V Jornadas de Enseñanza e Investigación Educativa en el campo de las Ciencias Exactas y Naturales. Universidad Nacional de La Plata

Pabón, J., Nieto, Z., y Gómez, C. (2015). Modelación matemática y GEOGEBRA en el desarrollo de competencias en jóvenes investigadores. . Revista Logos, Ciencia y Tecnología, 7(1), 65-70

Sánchez, A. (2012). Incorporación de las TICs en el aprendizaje de la matemática en el sector universitario. Revista De Educación Matemática, 27(3)

Suárez, P., Vélez, M., y Londoño, D. (2018). Las herramientas y recursos digitales para mejorar los niveles de literacidad y el rendimiento académico de los estudiantes de primaria. . Revista Virtual Universidad Católica del Norte, (54), 184-198

Suasnabas, L., Díaz, E., Ávila, W., y Rodríguez, V. (2017). Las Tics en los procesos de enseñanza y aprendizaje en la educación universitaria. Dom. Cien, 3(2), 721-749

Tamayo, E. (2013). Implicaciones didácticas de Geogebra sobre el aprendizaje significativo de los tipos de funciones en estudiantes de secundaria. . Apertura, 5(2), 58-69

Torres, P., y Cobo, J. (2017). Tecnología educativa y su papel en el logro de los fines de la educación. . Educere, 21(68), 31-40

Valderrama, J., y Saldaña, M. (2020). Influencia del softwareGeogebra enelrendimiento académico de los estudiantes delciclo Ide la EAPTurismo en el curso de Complemento Matemático-UNASAM,2017-I. Revista Pakamuros, 8(2), 77-84 
Zambrano, D., y Zambrano, M. (2019). Las tecnologías de la información y las comunicaciones (TIC's) en la educación superior: consideraciones teóricas. . Revista Electrónica Formación y Calidad Educativa (REFCalE), 213-228
Zenteno, F., Rivera, T., y Pariona, D. (2020). Tratamiento de las medidas de dispersión por medio del software GeoGebra. . Universidad y Sociedad, 12(1), 244-250 